\title{
Cubosome topologies at various particle sizes and crystallographic symmetries
}

\author{
W.T. Góźdź * \\ Institute of Physical Chemistry Polish Academy of Sciences, Kasprzaka 44/52, 01-224 \\ Warsaw, Poland \\ E-mail: wtg@ichf.edu.pl
}

\section{Supporting Information}

In the mean-field approximation the stable or metastable phases of the system correspond to the minimum of the functional (1).

$$
\mathcal{F}[\phi(\mathbf{r})]=\int d^{3} \mathbf{r}\left(|\triangle \phi(\mathbf{r})|^{2}+g[\phi(\mathbf{r})]|\nabla \phi(\mathbf{r})|^{2}+f[\phi(\mathbf{r})]\right) .
$$

In order to minimize the functional we discretize the field $\phi(\mathbf{r})$ on the cubic lattice. ${ }^{1}$ The first and the second derivatives in the gradient and Laplasian term of the Landau-Brazovskii functional at the point $\mathbf{r}=(i, j, k) h$ on the lattice were calculated according to the following formulas $^{2}$

$$
\frac{\partial \phi(\mathbf{r})}{\partial x} \rightarrow \frac{\phi_{i+1, j, k}-\phi_{i-1, j, k}}{2 h}
$$

and

*To whom correspondence should be addressed 


$$
\frac{\partial^{2} \phi(\mathbf{r})}{\partial x^{2}} \rightarrow \frac{1}{12 h^{2}}\left(-\phi_{i+2, j, k}+16 \phi_{i+1, j, k}-30 \phi_{i, j, k}+16 \phi_{i-1, j, k}-\phi_{i-2, j, k}\right),
$$

and similar in $y$ and $z$ directions.

The mixed derivatives are calculated ${ }^{2}$ according to

$$
\frac{\partial^{2} \phi(\mathbf{r})}{\partial x \partial y} \rightarrow-\frac{1}{2 h^{2}}\left(\phi_{i+1, j, k}+\phi_{i-1, j, k}+\phi_{i, j+1, k}+\phi_{i, j-1, k}-2 \phi_{i, j, k}-\phi_{i+1, j+1, k}-\phi_{i-1, j-1, k}\right)
$$

$\phi_{i, j, k}$ is the value of the field $\phi(\mathbf{r})$ at the point $\mathbf{r}=(i, j, k) h$, where $h$ is the lattice spacing. The calculation of derivatives on the lattice boundary requires taking into account the points outside the lattice. These points are given by appropriate boundary conditions.

After discretization, the problem of minimization of the functional is converted to the problem of minimization of a function of many variables, where the variables are the values of the field $\phi(\mathbf{r})$ at the lattice points. We use the conjugate gradient method to minimize the function numerically. $1,3,4$

In order to make the calculations for specific symmetry we can use appropriate kaleidoscopic cells. Two images in Fig. S1 show the field inside a cube ( on the right side) and in the smallest tetrahedral element (on the left side) obtained by the reduction of the volume inside the cube according to the symmetries of the space group Im3m. The color represent

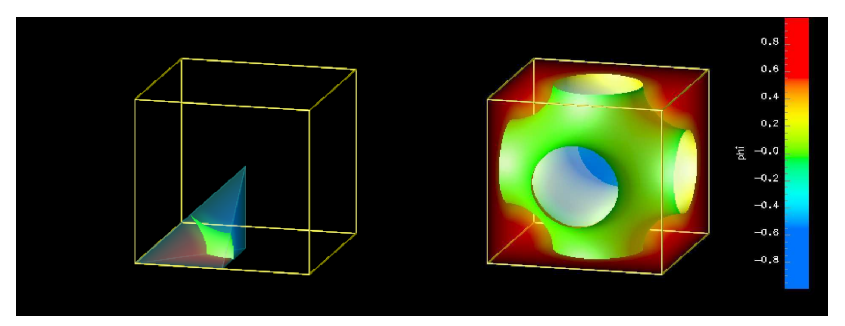

Figure S1: The field $\phi(\mathbf{r})$ inside the computational tetrahedron a cubic unit cell. Green and yellow colors show different sides of the surface $\phi(\mathbf{r})=0$.

the value of the field $\phi(\mathbf{r})$ at a given point in space. The surface colored by green and yellow 
is given by $\phi(\mathbf{r})=0$. The mapping of the field values to the color is done according to the colormap presented in Fig. S1. By exploiting the symmetry of the cubic phase it is possible to reduce the volume of the cube 48 times. Thus, the number of the variables in the function to be minimized can also be reduced. In an analogous way one can make calculations for cubic phases of other symmetries, but the computational polyhedra have different shape. ${ }^{1}$ When the priodic boundary conditions are applied we obtain infinite triply periodic bulk phases. For fixed boundary conditions we obtain closed objects with internal periodic structures.

The order parameter field $\phi(\mathbf{r})$ carries enormous amount of information about the local structure of the phases we have investigated. The most interesting is the topology of the phases, described by the surface $\phi(\mathbf{r})=0$. We use a simplified version ${ }^{1}$ of the marching cubes algorithm ${ }^{4}$ to determine the location of the surface $\phi(\mathbf{r})=0$. The surface $\phi(\mathbf{r})=0$ is given as a set of connected triangles.

The triangulated surface is used to calculate the Euler characteristics, $\chi$, of the surface inside the computational cell. The calculation of $\chi$ can be done according to the Euler formula $\chi=F+V-E$, where $\mathrm{F}, \mathrm{V}, \mathrm{E}$ is the number of faces $(\mathrm{F})$, vertices $(\mathrm{V})$, and edges (E) of the polygons cut out by the surface $\phi(\mathbf{r})=0$ in the small cubes of dimension of lattice spacing. The Euler characteristic for a closed surface is related to the Gaussian (K) curvature and the genus (g) of this surface in the following way ${ }^{5,6}$

$$
\chi=\frac{1}{2 \pi} \int_{S} K d S=2(1-g)
$$

where the integral is taken over the surface S. The genus is an integer number and describes how many holes are in a closed surface. For example the genus for a sphere is zero, for a torus is one and for a pretzel is two. The structures we have investigated are infinite and periodic. The genus for an infinite surfaces is infinite, of course, but for a finite piece of this surface, in a unit cell, is finite and characterizes the surface. Due to periodicity, the cuboidal 
cells can be treated as closed surfaces in four dimensions, making the calculation of the genus unambiguous ${ }^{7}$ Therefore, the genera of the structures inside the cuboids can be calculated according to: $g=1-\chi / 2$, where $\chi$ is the Euler characteristics for the surface inside the computational cell. The Gaussian and the mean curvatures are local characteristics of the internal surfaces given by $\phi(\mathbf{r})=0$. In the description of the model we have mentioned that some of the structures in the model should be characterized by zero mean curvature at every point of the internal interface. Here, we present the method used to compute Gaussian and mean curvatures. The unit normal $\mathbf{n}(\mathbf{r})$ at the point $\mathbf{r}$ is given by the gradient of the field $\phi(\mathbf{r})$ at the surface $\phi(\mathbf{r})=0$ :

$$
\mathbf{n}(\mathbf{r})=\frac{\nabla \phi(\mathbf{r})}{|\nabla \phi(\mathbf{r})|}
$$

The mean $(\mathrm{H})$ curvature is given by the divergence of the unit vector, ${ }^{8}$ normal to the surface at the point $\mathbf{r}, \mathbf{n}(\mathbf{r})$

$$
2 H(\mathbf{r})=-\nabla \cdot \mathbf{n}(\mathbf{r})
$$

and the Gaussian curvature $(\mathrm{K})$ by the formula ${ }^{9-11}$

$$
2 K(\mathbf{r})=\mathbf{n}(\mathbf{r}) \cdot \nabla^{2} \mathbf{n}(\mathbf{r})+[\nabla \cdot \mathbf{n}(\mathbf{r})]^{2}+[\nabla \times \mathbf{n}(\mathbf{r})]^{2}
$$

In the numerical calculations of the curvatures we used the following formulas: 8,12

$$
H=-\frac{1}{2 \sqrt{\phi_{x}^{2}+\phi_{y}^{2}+\phi_{z}^{2}}} \frac{B}{A}
$$




$$
K=\frac{1}{\phi_{x}^{2}+\phi_{y}^{2}+\phi_{z}^{2}} \frac{C}{A}
$$

where A, B , and C are obtained from:

$$
\operatorname{det}\left(\begin{array}{cccc}
\left(\phi_{x x}-\lambda\right) & \phi_{x y} & \phi_{x z} & \phi_{x} \\
\phi_{y x} & \left(\phi_{y y}-\lambda\right) & \phi_{y z} & \phi_{y} \\
\phi_{z x} & \phi_{z y} & \left(\phi_{z z}-\lambda\right) & \phi_{z} \\
\phi_{x} & \phi_{y} & \phi_{z} & 0
\end{array}\right)=A \lambda^{2}+B \lambda+C
$$

and are given by:

$$
\begin{gathered}
A=-\left(\phi_{x}^{2}+\phi_{y}^{2}+\phi_{z}^{2}\right) \\
B=\phi_{x}^{2}\left(\phi_{y y}+\phi_{z z}\right)+\phi_{y}^{2}\left(\phi_{x x}+\phi_{z z}\right)+\phi_{z}^{2}\left(\phi_{x x}+\phi_{y y}\right) \\
-2 \phi_{x} \phi_{y} \phi_{x y}-2 \phi_{x} \phi_{z} \phi_{x z}--2 \phi_{y} \phi_{z} \phi_{y z} \\
C=\phi_{x}^{2}\left(\phi_{y z}^{2}-\phi_{y y} \phi_{z z}\right)+\phi_{y}^{2}\left(\phi_{x z}^{2}-\phi_{x x} \phi_{z z}\right)+\phi_{z}^{2}\left(\phi_{x y}^{2}-\phi_{x x} \phi_{y y}\right) \\
+2 \phi_{x} \phi_{z}\left(\phi_{x z} \phi_{y y}-\phi_{x y} \phi_{y z}\right)+2 \phi_{x} \phi_{y}\left(\phi_{x y} \phi_{z z}-\phi_{x z} \phi_{y z}\right) \\
+2 \phi_{y} \phi_{z}\left(\phi_{y z} \phi_{x x}-\phi_{x y} \phi_{x z}\right)
\end{gathered}
$$

The mean and Gaussian curvatures have to be computed at the points of the surface $\phi(\mathbf{r})=0$. The derivatives of the field $\phi(\mathbf{r})$ at the point $\mathbf{r}_{0}$, for which $\phi\left(\mathbf{r}_{\mathbf{0}}\right)=0$, are calculated according to the formulas (2), (3), (4). 


\section{References}

(1) Góźdź, W. T.; Hołyst, R. Triply periodic surfaces and multiply continuous structures from the Landau model of microemulsions. Phys. Rev. E 1996, 54, 5012-5027.

(2) Abramowitz, M., Stegun, I. A., Eds. Handbook of Mathematical Functions With Formulas, Graphs, and Mathematical Tables, 9th ed.; National Bureau of Standards Applied Mathematics Series; U. S. Government Printing Office: Washington, D.C. 20402, 1970; Vol. 55; pages 883-884.

(3) Press, W. H.; Flannery, B. P.; Teukolsky, S. A.; Vetterling, W. T. Numerical Recipes; Cambridge University Press, 1990.

(4) Lorensen, W. E.; Cline, H. E. Marching cubes: A high resolution 3D surface construction algorithm. Computer Graphics 1987, 21, 163-169.

(5) Hoffman, D. A. Some basic facts, old and new, about triply periodic embedded minimal surfaces. Colloque de Physique 1990, 51 C7, 197-208.

(6) Anderson, D. M.; Davis, H. T.; Scriven, L. E.; Nitsche, J. C. C. Periodic surfaces of prescribed mean curvatures. Adv. Chem. Phys. 1990, 77, 337-396.

(7) Hyde, S. T. The topology and geometry of infinite periodic surfaces. Z. Kristallogr. 1989, 187, 165-185.

(8) Barnes, I. S.; Hyde, S. T.; Ninham, B. W. The caesium chloride zero potential surface is not the Schwarz P-surface. Colloque de Physique 1990, 51 C7, 19-24.

(9) Weatherburn, C., Ed. Differential Geometry Of Three Dimensions; At the University Press: Cambridge, 1930; Vol. II.

(10) Mackay, A. L.; Klimowski, J. Towards a grammar of inorganic structure. Comp. and Maths. with Appls. 1986, 12B, 803-824. 
(11) Gandy, P. J.; Bardhan, S.; Mackay, A. L.; Klinowski, J. Nodal surface approximations to the P,G,D and I-WP triply periodic minimal surfaces. Chemical Physics Letters 2001, $336,187-195$.

(12) Spivak, M. A Comprehensive Introduction to Differential Geometry; Publish or Perish: Berkley, 1979; Vol. III. 